\title{
What can NuSTAR do for X-ray bursts?
}

\author{
Jérôme Chenevez ${ }^{1}$ \\ DTU Space \\ Elektrovej 327,Kongens Lyngby, Denmark \\ E-mail: jerome@space.dtu.dk
}

\section{John Tomsick}

Space Sciences Laboratory, University of California, Berkeley, CA, 94720, USA

E-mail: jtomsick@ssl.berkeley.edu

\section{Deepto Chakrabarty}

Massachusetts Institute of Technology, 70 Vassar Street, Cambridge, MA 02139-4307, USA

E-mail: deepto@space.mit.edu

\section{Frits Paerels}

Columbia University, 1022 Pupin, 550 West 120th Street, New York, NY 10027, USA

E-mail:frits@astro.columbia.edu

\section{Finn E. Christensen}

DTU Space, Elektrovej 327, Kongens Lyngby, Denmark

E-mail: Finn@space.dtu.dk

\section{and The NuSTAR Team}

Unstable thermonuclear burning on the surface of accreting neutron stars is commonly observed as type I X-ray bursts. The flux released during some strong bursts can temporarily exceed the Eddington limit, driving the neutron star photosphere to such large radii that heavy-element ashes of nuclear burning are ejected in the burst expansion wind. We have investigated the possibility of observing with NuSTAR some X-ray bursters selected for their high burst rate and trend to exhibit so-called superexpansion bursts. Our main ambition is to detect the photoionization edges associated with the ejected nuclear ashes, and identify the corresponding heavy elements. A positive identification of such edges would probe the nuclear burning processes, and provide a measure of the expansion wind velocity as well as the gravitational redshift from the neutron star. Moreover, we expect that the high sensitivity of NuSTAR in hard $\mathrm{X}$-rays will make it possible to study the behaviour of the accretion emission during the bursts, which is an important parameter to constrain the properties of the X-ray burst emission and thermonuclear burning.

1

Speaker 
"An INTEGRAL view of the high-energy sky (the first 10 years)"

9th INTEGRAL Workshop and celebration of the 10th anniversary of the launch

October 15-19, 2012

Bibliotheque Nationale de France, Paris, France 


\section{Introduction}

X-ray bursters form a class of Low Mass X-Ray Binaries (LMXRBs) where accreted material from a donor star undergoes unstable thermonuclear burning in the surface layers of a neutron star. These type I X-ray bursts have a recurrence time of hours to days, which mainly depends on the accretion rate. Observations of X-ray bursts have made it possible to investigate the nuclear processing on the surface of neutron stars, leading to a better understanding of their inner thermal structure, magnetic field, and spin (see [1], for a review). The flux released can temporarily exceed the Eddington limit and drive the photosphere to large radii. Such photospheric radius expansion (PRE) bursts likely eject nuclear burning ashes into the interstellar medium, and may make possible the detection of spectral signatures from heavy elements [2].

The NuSTAR mission, successfully launched on $13^{\text {th }}$ June 2012, offers an unprecedented focusing view of the Universe in hard X-rays [3]. We propose to take advantage of the advanced sensitivity of the NuSTAR instrumentation to perform observations of X-ray bursters with the aim to detect spectral signatures, so as to study the physics of thermonuclear bursts up to hard X-rays.

In a recent paper, in 't Zand \& Weinberg [4] report on significant residuals in blackbody fits of superexpansion burst spectra obtained from RXTE observations. These authors interpret such residuals as absorption edge features consistent with photoionization signatures of Fe-peak elements. However, the relatively poor spectral resolution of these observations impedes the identification of these elements.

\subsection{Focusing on nuclear physics}

In some rare burst conditions, the peak luminosity has been observed to exceed the Eddington limit as much as the neutron star photosphere undergoes a superexpansion up to a radius of the order of $1000 \mathrm{~km}$ [4]. Such superexpansion bursts, characterised by the sudden and temporary disappearing of X-ray emission, are consistent with the burning of helium accumulated at low accretion rate, and are thus more likely to occur in Ultra-compact X-ray binaries (UCXBs), which contain an hydrogen-deficient white dwarf as donor companion [5, 6, 7]. The radiative winds produced by the burst can even eject in the interstellar medium the nuclear ashes from previous bursts, making it possible to detect absorption edges from heavy isotopes. Indeed, wind ejection models of Weinberg et al. [2] suggest that photoionization edges corresponding to the H-like ions of Fe-peak isotopes $\left({ }^{58} \mathrm{Fe}\right.$ at $9.2 \mathrm{keV},{ }^{59} \mathrm{Co}$ at $9.9 \mathrm{keV},{ }^{56} \mathrm{Ni}$ at $10.8 \mathrm{keV}{ }^{60} \mathrm{Zn}$ and ${ }^{62} \mathrm{Zn}$ at $12.2 \mathrm{keV}$ ) should be resolved by NuSTAR, for their equivalent width are predicted to exceed $600 \mathrm{eV}$.

Since the wind velocity is not expected to exceed $1 \%$ of the speed of light, relativistic shifting of the spectral signatures should not be an issue (N. Weinberg, personal communication), and a positive identification of the absorption edges would thus probe the nuclear burning. Moreover, if detected during the burst cooling tail, photoionization edges from heavy elements are expected to be observable from the base of the photosphere. This would uniquely provide a direct measure of the gravitational redshift at the surface of the neutron star, and thus constitute a probe of the ultra-dense matter equation-of-state. 


\section{Planned NuSTAR observations}

We have selected targets based on three criteria: 1) whether the source has exhibited spectral features consistent with being the diagnostic 9-13 keV absorption edges; 2) whether the source has a short enough burst recurrence time (at least in some states) to have a good chance of detecting one or more burst in a reasonable NuSTAR exposure; and 3) whether the source is known or suspected to be an UCXB system. The one target that satisfies all of these criteria is the archetypal UCXB $4 \mathrm{U}$ 1820-30, which, in its low state, has a burst recurrence time of a few hours. In fact, this source turns also to be the best candidate for displaying superexpansion bursts, and the low states recur at a periodicity of about 6 months [5].

The only other known or suspected UCXB to have a short enough recurrence time is $4 \mathrm{U}$ 1728-34 (aka GX 354-0, or the Slow Burster). A recent detection of a 10.77 minute periodic signal [10] provides good evidence for a He-rich donor. We propose target of opportunity observations of these two sources, so as to get the best chance to catch bursts. Exposures of 60 ks each should make possible to catch about $50 \mathrm{X}$-ray bursts per target.

\subsection{Simulations}

Figure 1 shows a NuSTAR simulation of an X-ray burst spectrum with an absorption edge at $8 \mathrm{keV}$. On this figure, Ri and Ro are respectively the expected incident and observed deadtime corrected count rates in the combined NuSTAR focal planes. The parameters for this simulation have been obtained for a superexpansion burst from $4 \mathrm{U}$ 1820-30 as described in [4], modelled by a $1.5 \mathrm{keV}$ absorbed black-body emission at the Eddington luminosity and redshifted edge at $8 \mathrm{keV}$ with optical depth $\tau=1$ during 10 seconds. Thanks to a lower background than RXTE, this simulation illustrates that NuSTAR will easily be able to identify such a photoionization edge during an X-ray burst.

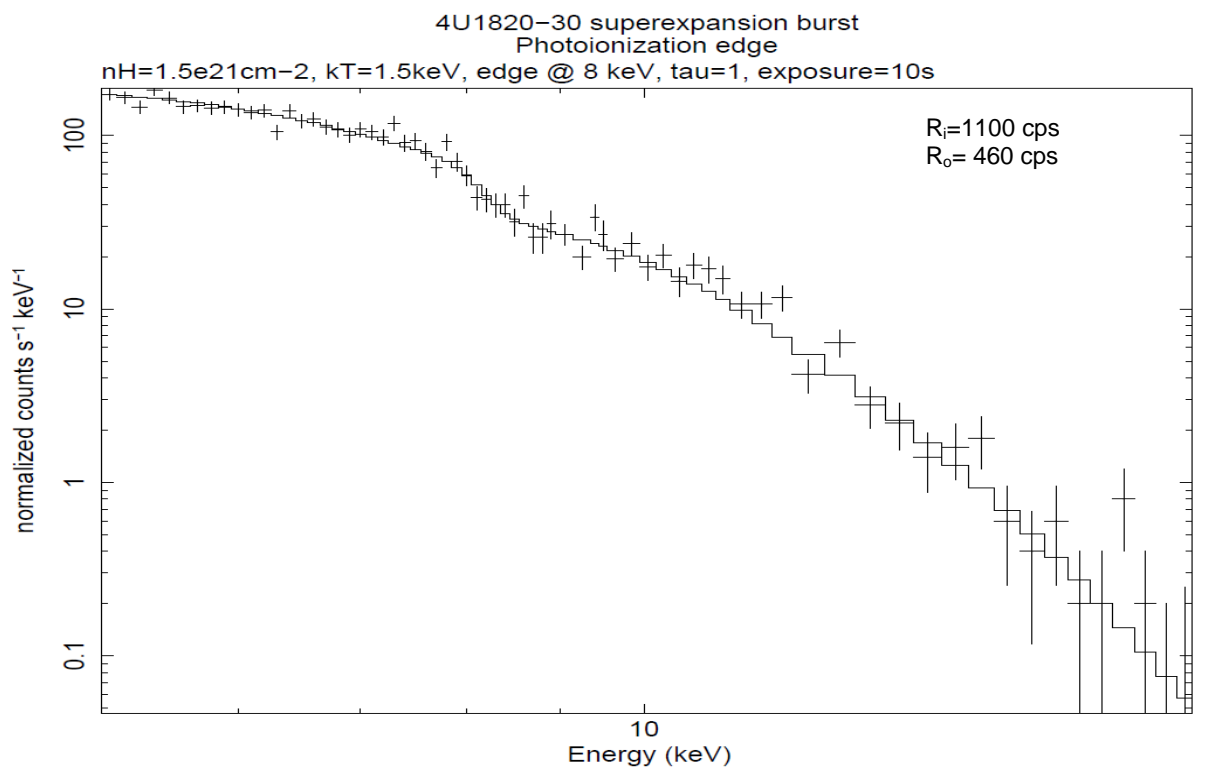

Figure 1: NuSTAR simulated X-ray burst spectrum with an absorption edge. 


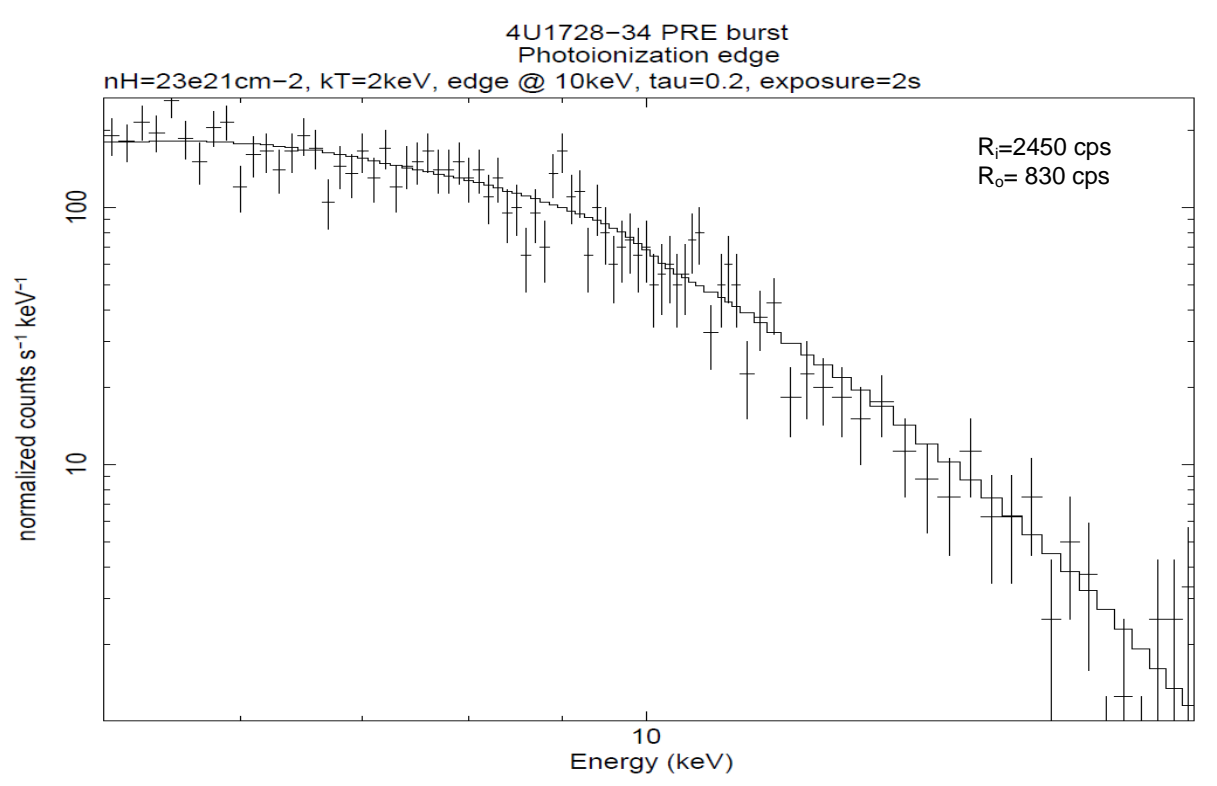

Figure 2: NuSTAR simulated spectrum of a moderate PRE X-ray burst.

Figure 2 shows a simulation example of a moderate photospheric radius expansion burst from $4 \mathrm{U}$ 1728-34 modelled by a $2 \mathrm{keV}$ absorbed black-body at the Eddington luminosity and an edge at $10 \mathrm{keV}$ with optical depth $\tau=0.2$ [10]. In this worst case, fitting the spectrum without the edge leads to $\chi^{2} / \mathrm{dof}=117.2 / 147$ against $\chi^{2} / \mathrm{dof}=114.9 / 145$ with the edge included in the fit; this marginal detection may be used as an upper limit on the optical depth.

\section{Additional science}

The accretion persistent emission of the bursting source, typically derived from flux measures in the last minutes prior to the burst, is often assumed as constant and subtracted from the total emission during a burst in order to obtain the net burst emission. However, variations of the "persistent" emission due to possible effects of the burst on the accretion disk should be taken into account, which is in practice not easy. Since the burst emission itself is mainly limited below $25-30 \mathrm{keV}$, and the persistent emission is consistent with higher energy processes, NuSTAR should therefore provide for the first time the possibility to separate these two components from each-other.

Moreover, likely observations of atomic X-ray spectral components reflected from the inner accretion disk have been reported [8,9]. The high spectral resolution capabilities of NuSTAR may allow us to differentiate between the potential interpretations of the X-ray bursts spectral features. 


\section{Summary}

We hope to catch with NuSTAR a superexpansion burst that would display photoionization edges. A successful detection of absorption edges during type I X-ray bursts will imply that heavy ions, nuclear ashes of the thermonuclear burning, are ejected from the neutron star. Our main objective is to confront the X-ray burst observational properties with the current theory in order to:

1. better understand the complexity of the relationship between nuclear burning and accretion process regimes,

2. probe the nuclear burning processes involved during X-ray bursts,

3. study the effects caused by the burst on the accretion disk around the neutron star.

\section{Acknowledgements}

JC acknowledges fruitful discussions with J. in 't Zand, D. Galloway, E. Kuulkers, S. Brandt, H. Schatz, and is grateful to the International Space Science Institute in Bern, where part of this work was completed. This work was supported by ESA-PRODEX contract N: 90057.

\section{References}

[1] T. Strohmayer \& L. Bildsten, 2006 [astro-ph/0301544]

[2] N. Weinberg, L. Bildsten, and H. Schatz, ApJ 639, 1018, 2006.

[3] F.A. Harisson.et al., SPIE 7732, 77320S, 2010.

[4] J. in 't Zand \& N. Weinberg, A\&A 520, 81, 2010.

[5] J. in 't Zand et al., A\&A 547, 47, 2012.

[6] A. Cumming, ApJ 595, 1077, 2003.

[7] J. in 't Zand, P. Jonker, and C. Markwardt, A\&A 465, 953, 2007.

[8] T. Strohmayer \& E. Brown, ApJ 566, 1045, 2002.

[9] E. Kuulkers, et al., A\&A 399, 663, 2003.

[10] D. Galloway et al., ApJ 724, 417, 2010. 